\title{
Clinical Medical Education: Cardiothoracic Surgery in the Era of COVID-19
}

\author{
Andy Dong ${ }^{1}$, Francis Simpson ${ }^{1}$, William $Q u^{1}$, and Jeffrey Javidfar ${ }^{1}$ \\ ${ }^{1}$ Emory University School of Medicine
}

September 10, 2020

\begin{abstract}
Background and Aim: Clinical education has been disrupted by the COVID-19 pandemic. We present a standardized remote alternative online cardiothoracic surgery primer to accommodate a shortened clinical calendar. Methods: A week-long cardiothoracic surgery course consisting of virtual case-based lectures and small groups as well as surgical operation walkthroughs was conducted iteratively through April and May 2020 at Emory University School of Medicine, Atlanta, GA for new clinical third-year medical students. Results: Remote learning platforms helped maintain medical student clinical education. Cardiothoracic procedure video walkthroughs were highly demanded for remote learning. Virtual small group discussions were felt to be invaluable in facilitating active problem solving and clinical decision making of cardiothoracic surgery. Conclusion: Our online cardiothoracic surgery curriculum can be a framework for alternative medical student clinical education. Student feedback is necessary as we adapt to teaching during the COVID-19 pandemic and future global disruptions.
\end{abstract}

Title: Clinical Medical Education: Cardiothoracic Surgery in the Era of COVID-19

Authors: Andy Dong, BS ${ }^{1}$, Francis Simpson, MS ${ }^{1}$, William Qu, MD², Jeffrey Javidfar, MD²

Affiliation:

1. Emory University School of Medicine

2. Division of Cardiothoracic Surgery, Department of Surgery, Emory University School of Medicine

Disclosure: The authors do not have a conflict of interest. No funding was used for this paper.

Word Count: 495

Corresponding Author:

Jeffrey Javidfar, MD

The Emory Clinic

1365 Clifton Road NE

Building A, Suite 2220

Atlanta, Georgia 30322

Phone: (404) 778-3730

Jeffrey.Javidfar@emory.edu

The call to action by Newman and Lattouf echoed the concerns of many educators and students for the need of curricula that adequately support clinical education during COVID-19. ${ }^{1,2,3}$ With the possibility of COVID-19 cases increasing in the fall, third- and fourth-year clerkships may become remote. Furthermore, 
clinical students have resumed the semester with modified and shortened schedules which may curtail exposure to "non-essential" electives like cardiothoracic surgery. Thus, we present our virtual cardiothoracic surgery curriculum and remote teaching experience with clinical-year medical students. Our aim is to help maintain clerkship education with an alternative distance-learning option to accommodate a compressed clinical calendar impacted by COVID-19.

We created a week-long cardiothoracic surgical education course focusing on interactive small group lectures and discussions as well as surgical operation walkthroughs (Figure 1). Although concise, we felt a week-long course was an appropriate amount of time to cover a wide array of cardiothoracic topics given it is 25-50\% the length of a surgical subspecialty rotation. Generally, the day started with virtual case lectures via Zoom (Zoom Video Communications Inc., San Jose, CA) followed by supplemental pre-recorded video didactics, then small group discussions in the afternoon. Paramount to clinical education is learning clinical problem solving and decision making. Sessions were interactive with a maximum of five students in order to easily facilitate active conversation and case-based problem solving. Faculty also walked students through surgical cases during the aforementioned small group lectures and discussions. Procedure videos and case-based discussions provided a welcomed contrast to didactic teaching that allowed students to participate in active clinical decision making. Content-wise, we focused on main cardiothoracic diseases and surgeries (Figure 1) with special attention drawn toward extracorporeal membrane oxygenation (ECMO) and its significance as a treatment modality for severe acute respiratory distress syndrome for COVID-19. ${ }^{5}$

We taught this course over April and May 2020 to new third-year medical students. We received survey feedback from every student at the end of each week and made continuous iterative adjustments. Students cited the benefit of a smaller, more interactive class with a single primary instructor, particularly highlighting the afternoon small group discussions and case problem solving. They also appreciated the contemporary relevance of the ECMO series. The main critique was that students really wanted more surgical videos, and so we integrated more surgical footage into our discussions. From our survey, students expressed an increase in understanding and interest in cardiothoracic surgery. Preliminary analysis of the first ten preand post-assessment results demonstrated an increase in mean scores from $62 \%$ to $80 \%$. For future course considerations, suture skills sessions could also be incorporated in the course to provide a more handson experience (though this requires safe distribution of suture materials). Small group discussions were invaluable, and future courses should continue to integrate active problem solving and case management pertinent to clerkship students.

As the pandemic rattles the framework of medical education, we hope that our remote learning curriculum can be utilized at other medical schools for cardiothoracic clerkship education.

\section{References}

1. Newman NA, Lattouf OM. Coalition for medical education-A call to action: A proposition to adapt clinical medical education to meet the needs of students and other healthcare learners during COVID-19. $J$ Card Surg. 2020.

2. Rose S. Medical Student Education in the Time of COVID-19.JAMA. 2020.

3. Theoret C, Ming X. Our Education, Our Concerns: Medical Student Education Impact due to COVID-19. Med Educ. 2020.

4. MacLaren G, Fisher D, Brodie D. Preparing for the Most Critically Ill Patients With COVID-19: The Potential Role of Extracorporeal Membrane Oxygenation. JAMA. 2020;323:1245-1246.

\section{Hosted file}

Letter to the Editor Figure.docx available at https://authorea.com/users/355388/articles/

478640-clinical-medical-education-cardiothoracic-surgery-in-the-era-of-covid-19 